CASE REPORT-

Volume 16 Issue 22021

DOI: 10.21315/aos2021.16.2.14

ARTICLE INFO

Submitted: 02/40/2021

Accepted: 24/06/2021

Online: 22/12/2021

\section{An Unusual Early Oral Presentation of Acromegaly: A Case Report}

\author{
Ayat Gamal-AbdelNaser
}

Department of Oral Medicine and Periodontology, Faculty of Dentistry, Cairo University, Cairo 11562, Egypt

E-mail: ayat.gamal@dentistry.cu.edu.eg

To cite this article: Gamal-AbdelNaser A (2021). An unusual early oral presentation of acromegaly: A case report. Arch Orofac Sci, 16(2): 253-258. https://doi.org/10.21315/aos2021.16.2.14

To link to this article: https://doi.org/10.21315/aos2021.16.2.14

\begin{abstract}
Acromegaly is a devastating chronic slowly progressive disease. Its early diagnosis is a challenging issue that necessitates clinical suspicion of signs and symptoms as a first step. This report introduces an unusual early sign in the oral cavity that lead to the early diagnosis of an acromegaly case. A case of a healthy 40-year-old male patient presented with progressively growing multiple hard swellings in the upper and lower jaws. Clinical examination revealed bony hard multiple small spiky exostosis-like swellings, located at the maxillary and mandibular alveolar bones. An array of investigations revealed a 2-mm diameter pituitary tumour in MRI of sella. To the best of the author's knowledge, this is the first report of spiky exostosis-like growths in the alveolar bone as an early sign of acromegaly. In this case, thorough examination of oral signs and symptoms was the first step for early diagnosis and hence, better prognosis for acromegaly.
\end{abstract}

Keywords: Early detection; exostosis; growth hormone; oral diagnosis; pituitary gland

\section{INTRODUCTION}

Acromegaly is an endocrinal disorder caused by oversecretion of growth hormone $(\mathrm{GH})$ from the pea-sized pituitary gland (AlDallal, 2018). The excessive secretion of GH causes deleterious effects on almost all the body systems over long period of time (Katznelson et al., 2011). The symptoms range between continuous enlargement of soft tissues due to increase in mucopolysaccharide deposition, continuous subperiosteal bone formation enlarging the acral and facial bones, and severe life threatening systemic conditions. Being the most apparent manifestations, facial and oral symptoms include thick lips, macroglossia with lateral indentations, prognathism causing Class III type of occlusion, spacing of teeth and anterior open bite, major salivary gland enlargement and coarsening of facial skin and features (Katznelson et al., 2011; Atreja et al., 2012). However, the disease has a slow insidious onset which delays its diagnosis for about 10-12 years. Therefore, early diagnosis is considered a challenge for endocrinologists as it would spare the patients severe systemic involvement and premature death (AlDallal, 2018). In this report, the disease was diagnosed based on an unusual presentation detected by an oral medicine specialist.

\section{CASE REPORT}

This case was managed after the patient's informed consent and in accordance with the ethical standards of ethics committee of Faculty of Dentistry-Cairo University, and with the Declaration of Helsinki and 
its revision. This report presents a case of a medically healthy 40-year-old male patient who presented to the dental clinic complaining of pain in the gums arising from hard, sharp, pointed swellings in the upper and lower jaws. The patient reported the swellings to grow progressively since it first appeared one year earlier. The swellings were noticed to be related to the jawbone at the labial aspect of the lower incisors and at the buccal aspects of the upper molar areas bilaterally.

Examination revealed bony hard multiple round small swellings, ranging in diameter between $0.2 \mathrm{~cm}$ and $1 \mathrm{~cm}$, located at the labial aspect of the mandibular alveolar bone related to the root of each of the lower anterior teeth. The mucosa covering the swellings was normal-looking and tender to pressure. Similar lesions were detected at the labial and buccal aspects of the maxillary alveolar bone. The lesions were recognised to be more pronounced posteriorly bilaterally forming a shelf of bone periapical to the molar area. A solitary similar lesion was seen palatal to the missing upper left second molar with diameter of $2 \mathrm{~cm}$. The alveolar ridge at the site of missing upper left first and second molars was apparently broad. The teeth related to the sites of swelling were examined and found to be clinically sound, non-tender to percussion and periodontally healthy. Anterior cross bite was detected, however unrecognised or complained of by the patient. Otherwise, no other abnormalities were detected in the oral mucosa or the tongue (see Fig. 1).

Upon examination, the patient suffered from breathlessness when the back was declined at supine position. Furthermore, extraoral examination revealed a bit pronounced facial features and spade-like hands (see Fig. 2). Differential diagnosis included bone exostosis, multiple expansile intrabony lesions, Paget's disease of bone and acromegaly. Therefore, cone beam computed tomography (CBCT) was primarily requested to exclude intrabony lesions. It showed normal bone density and trabeculation. However, it revealed spiky projections of bone at the buccal and palatal alveolar bones of the upper teeth, mostly pronounced at the molar region bilaterally (see Fig. 3).

Furthermore, laboratory investigations showed normal levels of serum calcium (9.5 $\mathrm{mg} / \mathrm{dl})$, phosphorus $(2.9 \mathrm{mg} / \mathrm{dl})$
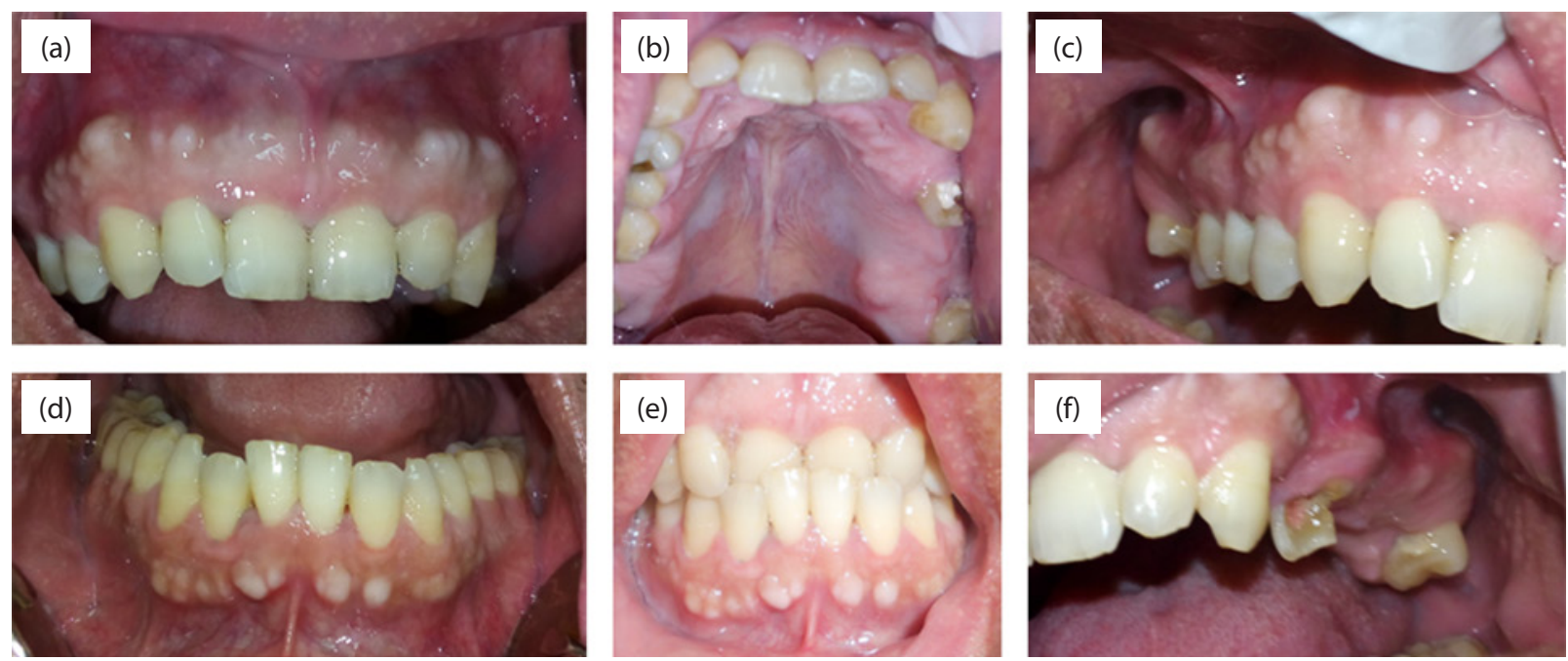

Fig. 1 The intraoral presentation of the case. (a) and (d) showing the bony swellings at the labial aspects of the maxillary and mandibular alveolar bones; (b) shows the exostosis at the palatal plate of alveolar bone;

(c) and (f) show the spiky pattern of the swellings at the right and left buccal alveolar bones; and (e) shows the anterior cross bite. 

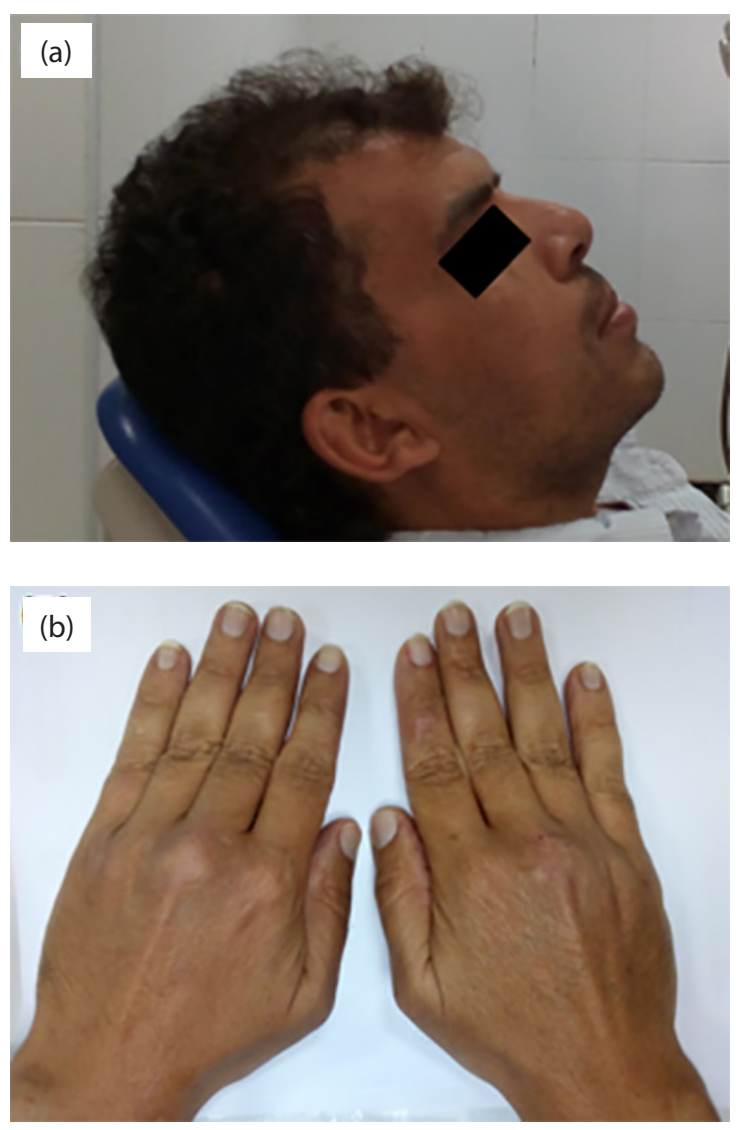

Fig. 2 The extraoral presentation of the case. (a) shows the facial features; and

(b) clarifies the spade-like hands.

and alkaline phosphatase (109 IU/L). Therefore, Paget's disease of bone was excluded. Thus, the patient was referred to an endocrinologist who requested tests for growth hormone $(\mathrm{GH})$ action: Insulin like growth factor-1 (IGF-1) and oral glucose tolerance test (OGTT) for $\mathrm{GH}$ suppression. IGF-1 level was within normal range $(127 \mathrm{ng} / \mathrm{ml})$ while less-thannormal response was detected to OGTT (basal $=0.4 \mathrm{ng} / \mathrm{ml}, 1 / 2 \mathrm{~h}=0.1 \mathrm{ng} / \mathrm{ml}$, $1 \mathrm{~h}=0.1 \mathrm{ng} / \mathrm{ml}, 11 / 2 \mathrm{~h}=0.2 \mathrm{ng} / \mathrm{ml}$, $2 \mathrm{~h}=0.5 \mathrm{ng} / \mathrm{ml}$ ). Therefore, MRI to sella was performed, showing a space-occupying lesion at the right side of the pituitary of maximum dimensions of $1 \times 2 \mathrm{~mm}$, with normal picture of the optic chiasma, optic nerve and brain. The findings suggested right-sided pituitary micro-adenoma (see Fig. 4).
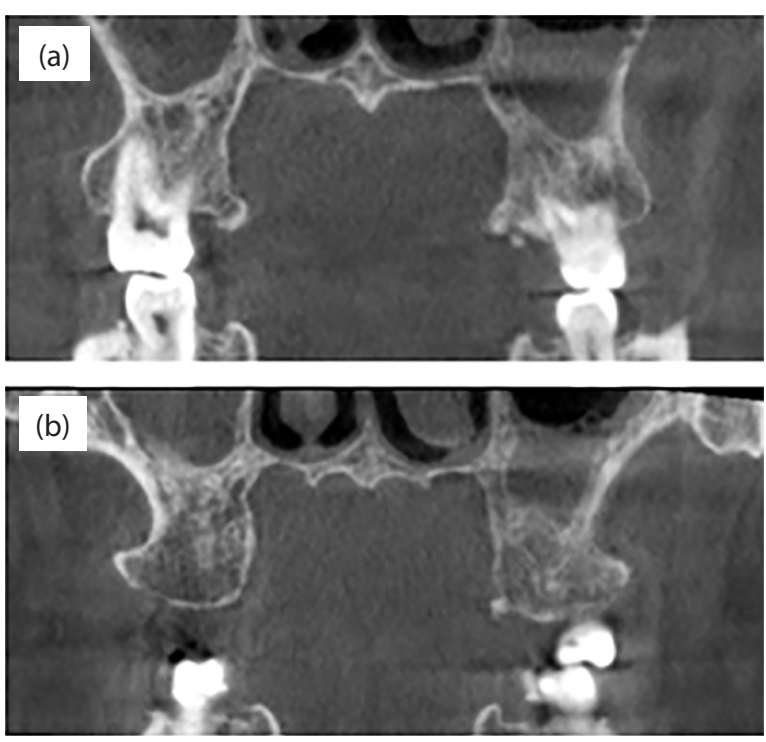

Fig. 3 Two coronal cuts of the CBCT at the (a) last molar region; and (b) first molar region revealing the spiky pattern of the bone exostosis at the buccal and palatal plates of bone related to the molar region of the maxilla bilaterally.

Furthermore, laboratory investigations were performed to detect the general condition of the body and the other functions of the pituitary gland. The serum levels of other pituitary hormones were all within normal range (Tri-iodothyronine $(\mathrm{T} 3)=1.25 \mathrm{ng} / \mathrm{ml}$, Thyroxine $(\mathrm{T} 4)=8.13 \mathrm{ug} / \mathrm{dl}$, Thyroid stimulating hormone $(\mathrm{TSH})=1.41 \mathrm{uIU} / \mathrm{mL}$, Prolactin $=8.63 \mathrm{ng} / \mathrm{ml}$, Follicle stimulating hormone $(\mathrm{FSH})=5.56 \mathrm{mlU} / \mathrm{Ml}$, Luteinizing hormone $(\mathrm{LH})=6.02 \mathrm{mlU} / \mathrm{Ml}$, Adrenocorticotropic hormone (ACTH) $=14.82 \mathrm{pg} / \mathrm{ml}$ ). Also, liver and kidney functions were normal, together with blood glucose level. On the other hand, complete blood picture showed polycythemia. Finally, diagnosis was confirmed to be acromegaly due to pituitary tumour.

As a second step after pituitary tumour was detected, visual field examination was performed, showing visual field defect in the right eye. A neuro-surgery was indicated for excising the pituitary tumour and a minor oral surgery was indicated for alveoloplasty of the sharp bony edges. 

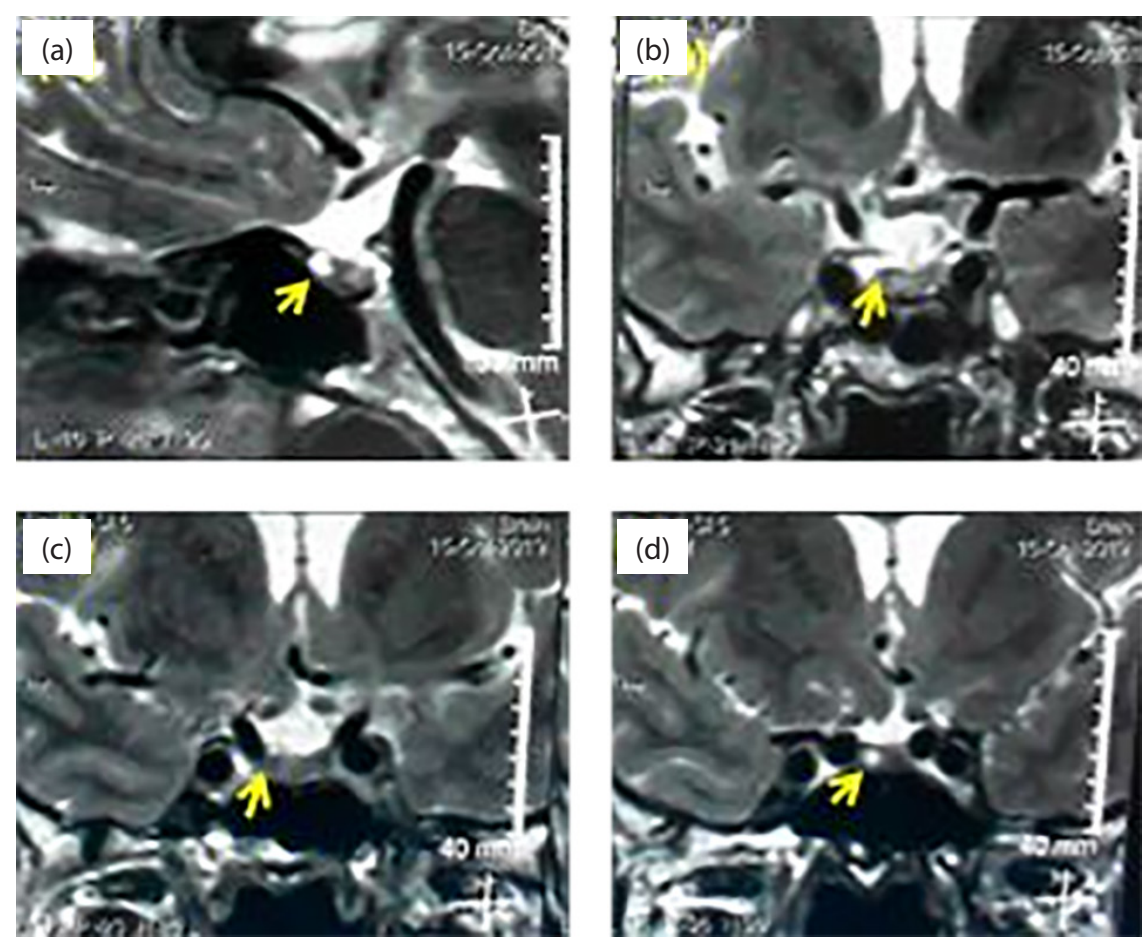

Fig 4. Contrast enhanced MRI of the sella tursica. (a) Showing a sagittal section with the yellow arrowhead pointing to the enhanced picture of the pituitary adenoma; (b), (c) and (d) showing sequential coronal cuts of the sella with yellow arrow heads indicating the site of the lesion at the right side of the pituitary.

\section{DISCUSSION}

The pituitary gland, seated at the sella tursica, is responsible for releasing a wide array of hormones. When a tumour affects the pituitary somatotroph cells, it oversecretes GH. These high levels of GH stimulate some organs, mainly the liver, to produce high levels of IGF-1 in blood. Consequently, IGF-1 affects almost all the body organs and systems causing soft tissue and acral enlargement, joint pain, hypertension, diabetes mellitus and most gravely cardiac and respiratory failure. All these effects increase the mortality rates of patients having active acromegaly to 2-4 times higher than that of the general population (Lugo et al., 2012; AlDallal, 2018).

Beside the somatic effects of acromegaly induced by the high levels of $\mathrm{GH}$, the expanding pituitary tumour further induces local effects in the form of headaches and visual field defects due to compression of the optic chiasma (AlDallal, 2018). Therefore, early diagnosis of acromegaly is crucial to stop the disease process as early as possible. However, the slowly progressive course of the disease causes slow alterations that are generally ignored by the patient, surroundings and sometimes physicians. Therefore, clinical suspicion plays the major role in diagnosis. Only if a clinician suspects acromegaly, that biochemical investigations and imaging would be performed, and eventually the case would be successfully diagnosed and treated (AlDallal, 2018).

Biochemical diagnostic investigations of acromegaly do not rely on testing serum GH levels due to its physiologically fluctuating levels throughout the day. Thus, the testing process starts by investigating the IGF-1 level. However, the results can be altered by multiple physiological factors; moreover, the technique suffers major technical problems. Therefore, plasma GH level after OGTT 
represents the gold standard for diagnosis of acromegaly (Cordido et al., 2013; AlDallal, 2018). Yet, the method of the assay may also impact the results of GH levels after OGTT. So, if performed solely, biochemical tests may cause the diagnosis to be missed (Zahr \& Fleseriu, 2018). As a last resort, whenever the biochemical tests and clinical picture arise suspicion, pituitary MRI is requested. It provides sensitive and reliable results that may identify the smallest tumours, tumour invasiveness and its compression of the surrounding structures (Cordido et al., 2013; Katznelson et al., 2014; AlDallal, 2018).

After successful diagnosis, treatment options include surgical excision of the tumour, medical therapy, and/or radiation therapy (Cordido et al., 2013; AlDallal, 2018). In conclusion, the major obstacle in acromegaly case management is the delayed diagnosis. A vital step to tackle this obstacle is the higher awareness about the disease signs and symptoms among primary healthcare physicians, dentists and other healthcare professionals (Katznelson et al., 2011; AlDallal, 2018). After a thorough literature search, articles reporting oral symptoms in acromegaly patients only include reports of previously diagnosed patients whose oral, dental and occlusal records are being documented (Herrmann et al., 2011; Kashyap et al., 2011). Hence, to the best of our knowledge, this is the first report where an oral medicine specialist spotted an acromegaly patient based on oral symptoms. Furthermore, no previous report ever stated progressive exostosis-like bone formation in the alveolar bones of the jaws as a symptom of acromegaly.

The mechanism of increased bone thickness in acromegaly has been previously intensely studied and explained. High serum levels of IGF-1 are proven to stimulate osteoblasts to actively increase periosteal bone deposition, thereby, increase the cortical bone mass. In the oral cavity, this action is known to cause a generalised enlargement of the mandible, manifesting in the form of prognathism with Class III type of malocclusion (Mazziotti et al., 2019).
In this report, we propose that the periosteal bone formation in the jaw bones of acromegaly patients may not only be in a symmetrical form to the whole bone causing the characteristic prognathism. It may also include a sporadic manner of bone deposition in an exostosis-like pattern, but with a rather progressive course and spiky pattern. The case of the hereby presented patient was suspected to be acromegalic when shortness of breath was observed upon lying in more supine positions. The acral and facial features were not very conclusive. Despite of having anterior cross bite and extraoral symptoms, the patient was only concerned with the painful sharp bony swellings rather than the asymptomatic slowly progressing facial and acral changes. Together with the absence of any available reports of similar oral findings, acromegaly was not on the top of the differential diagnosis list.

Bone exostosis as a normal variant was on the top of the list. But it is not reported to have progressively growing course in adults. Furthermore, palatal bone is not a favoured site for bone exostosis (Kerr et al., 2015). Therefore, it was excluded. Also, the second diagnosis on the list, namely multiple expansile intrabony lesions, was excluded by CBCT. The next suspected diagnosis was Paget's disease of bone. But, it was excluded after the serum alkaline phosphatase level was found to be normal, compared to the extensively high levels in Paget's disease of bone (Kerr et al., 2015). Therefore, a referral to an endocrinologist was performed to undergo the essential investigations for acromegaly. Diagnosis was confirmed by MRI to be acromegaly due to microadenoma in the pituitary gland. Furthermore, visual field defect was detected.

The current report clarifies that the patient already had other acromegaly signs including anterior crossbite, facial and acral alterations. However, all these signs and symptoms have been missed or ignored by the patient due to being slowly progressive and asymptomatic. However, the multiple small sharp pointed 
bone overgrowths in the jaw bones overstretched and pricked the overlying mucosae, causing pain. Luckily, this pain was the only motive for the patient to seek medical help by a dental practitioner.

This report endorses the recommendations of the guidelines of the American Association of Clinical Endocrinologists regarding educating other medical professionals including dentists about the signs and symptoms of acromegaly (Katznelson et al., 2011). The report introduces the author's experience as a living proof of the crucial importance of dentist's thorough examination, suspicion and referral in early diagnosis and improving disease prognosis. It is not a cliché that dental professionals may be the first to see the signs.

\section{CONCLUSION}

Acromegaly may manifest as spiky exostosislike bone growths in the alveolar bone. Hence, thorough routine comprehensive intraoral examination should be linked to the general clinical condition of the patient. Together, these upraise clinical suspicion that can be the first step for early detection of the pituitary disease; improving its prognosis.

\section{REFERENCES}

AlDallal S (2018). Acromegaly: A challenging condition to diagnose. Int $\mathcal{F} \mathrm{Gen} \mathrm{Med,} \mathrm{11:}$ 337-343. https://doi.org/10.2147/IJGM .S169611

Atreja G, Atreja SH, Jain N, Sukhija U (2012). Oral manifestations in growth hormone disorders. Indian f Endocrinol Metab, 16(3): 381-383. https://doi.org/10.4103/2230 $-8210.95678$

Cordido F, García Arnés JA, Marazuela Aspiroz M, Torres Vela E (2013). Practical guidelines for diagnosis and treatment of acromegaly. Endocrinol Nutr, 60(8): 457.e1457.e15. https://doi.org/10.1016/j.endoen .2013 .10 .012
Herrmann BL, Mortsch F, Berg C, Weischer T, Mohr C, Mann K (2011). Acromegaly: A cross-sectional analysis of the oral and maxillofacial pathologies. $\operatorname{Exp}$ Clin Endocrinol Diabetes, 119(1): 9-14. https://doi.org/10.1055/s-0030-1255020

Kashyap RR, Babu GS, Shetty SR (2011). Dental patient with acromegaly: A case report. f Oral Sci, 53(1): 133-136. https://doi.org/ 10.2334/josnusd.53.133

Katznelson L, Atkinson JL, Cook DM, Ezzat SZ, Hamrahian AH, Miller KK et al. (2011). American Association of Clinical Endocrinologists Medical Guidelines for clinical practice for the diagnosis and treatment of acromegaly - 2011 update: Executive summary. Endocr Pract, 17(4): 636-646. https://doi.org/10.4158/EP.17.4 .636

Katznelson L, Laws ER, Melmed S, Molitch ME, Murad MH, Utz A et al. (2014). Acromegaly: An endocrine society clinical practice guideline. $\mathcal{f}$ Clin Endocrinol Metab, 99(11): 3933-3951. https://doi.org/ 10.1210/jc.2014-2700

Kerr AR, Chan KC, Phelan JA (2015). Benign lesions of the oral cavity and the jaws. In: Glick M (ed.), Burket's Oral Medicine, 12th edn. Shelton: People's Medical Publishing House. pp. 147-172.

Lugo G, Pena L, Cordido F (2012). Clinical manifestations and diagnosis of acromegaly. Int $\mathcal{F}$ Endocrinol, 2012: 540398. https://doi .org/10.1155/2012/540398

Mazziotti G, Lania AGA, Canalis E (2019). Bone disorders associated with acromegaly: Mechanisms and treatment. Eur $\mathcal{f}$ Endocrinol, 181(2): R45-R56. https://doi .org/10.1530/EJE-19-0184

Zahr R, Fleseriu M (2018). Updates in diagnosis and treatment of acromegaly. Eur Endocrinol, 14(2): 57-61. http://doi.org/10 $.17925 /$ EE.2018.14.2.57 